\title{
HEREDITARINESS OF STRONG AND STABLE RADICALS
}

\author{
by E. R. PUCZYŁOWSKI
}

(Received 2 September, 1980)

The aim of this paper is to discuss some relations among hereditary, strong and stable radicals. In particular we investigate hereditariness of lower strong and stable radicals. Some facts obtained are related to some results and questions of $[2,6,7]$.

All rings in the paper are associative. Fundamental definitions and properties of radicals may be found in [9]. Definitions of hereditary and strong radicals are used as in Sands [7]. We say that a radical $S$ is left (right) stable if

$(\rho)$ : for every ring $R$ and every left (right) ideal $I$ of $R$ it follows $S(I) \subseteq S(R)$. Of course any left (right) stable radical is left (right) strong.

1. General remarks. In what follows $M$ will denote a homomorphically closed class of rings and $L M(S M, S t M)$ will be the lower (lower left strong [1], lower left stable [4]) radical determined by $M$.

For any ring $A, A^{0}$ denotes the zero-ring on the additive group of $A$. For any class $M$ we define $M^{0}=\left\{A \mid A^{0} \in M\right\}$. It is easy to see that if $M$ is a radical then so is $M^{0}$. If a radical $M$ is hereditary and left (right) strong then $M \subseteq M^{0}$ (cf. [7]).

If $P$ is a subring of a ring $R$ then $P^{*}$ will denote the ideal of $R$ generated by $P$. Of course $P^{*}=R^{1} P R^{1}$ where $R^{1}$ is the natural extension of $R$ to the ring with unity.

Lemma 1. Let $S$ be a radical and $P$ a subring of a ring $R$ such that $P^{0} \in S$. Then $\left(P^{*}\right)^{0} \in S$. In particular if $S$ is left strong (stable) then so is $S^{0}$.

Proof. Since $P^{*}=R^{1} P R^{1}$ then $\left(P^{*}\right)^{0}=\sum_{a, b \in R^{1}}(a P b)^{0}$. Now it is easy to see that for any $a, b \in R^{1}, f: P^{0} \rightarrow(a P b)^{0}$ given by $f(p)=a p b$ is a ring homomorphism, so $(a P b)^{0} \in S$. Thus $\left(P^{*}\right)^{0} \in S$.

Corollary 1. If $M \subseteq M^{0}$ then $L M \subseteq(L M)^{\circ}, S M \subseteq(S M)^{\circ}$ and $S t M \subseteq(S t M)^{\circ}$.

Proof. Since $M \subseteq L M$ and $M \subseteq M^{0}$ then $M \subseteq(L M)^{0}$. But $(L M)^{0}$ is a radical, so $L M \subseteq(L M)^{0}$. Analogously, using Lemma 1 , we obtain the result for $S M$ and $S t M$.

For any class $M$ let us define

$$
\bar{M}=\{A \in M \mid \text { every ideal of } A \text { is in } M\} \text {. }
$$

It is easy to see that for any radical $S, \bar{S}$ is also radical. If $M=\bar{M}$ then $M=\bar{M} \subseteq \overline{L M}$, so $L M=\overline{L M}$. Thus we have the well known

COROLLARY 2. The lower radical determined by a hereditary class is hereditary.

Remark. Analogously as above, if $\sigma$ is an $H$-relation in the sense of [5] and $S$ is a radical then $\bar{S}=\{A \in S \mid$ if $B \sigma A$ then $B \in S\}$ is also radical. Thus if $M$ is $\sigma$-hereditary then

Glasgow Math. J. 23 (1982) 85-90. 
so is $L M$. This proof is only somewhat simpler than that of [5], but this approach is very convenient when we investigate hereditariness of lower strong or stable radicals.

Proposition 1. If a radical $S$ has the properties (a) $S$ is left strong, (b) $S \subseteq S^{0}$, and (c) zero-rings from $S$ are in $\bar{S}$, then $\bar{S}$ is left strong.

Proof. Let $L \in \bar{S}$ be a left ideal of $A$ and $I$ an ideal of $L^{*}$. Then $I L$ is an ideal of $L$ and a left ideal of $I$. Thus $I L \subseteq S(I)$. Now $I\left(L^{*}\right)^{2}=I L A^{1} L^{*}=I L L^{*} \subseteq S(I) L^{*} \subseteq S(I)$. In particular $I^{3} \subseteq I\left(L^{*}\right)^{2} \subseteq S(I)$. This implies that $\left(I^{2}+S(I)\right) / S(I)$ is isomorphic to some ideal of $\left(L^{*} / S(I)\right)^{0}$. Now the assumptions (a) and (b) give $\left(L^{*} / S(I)\right)^{0} \in S$, so by (c) $\left(I^{2}+S(I)\right) / S(I) \in S$. But then $I^{2} \subseteq S(I)$. Thus $I / S(I)$ is a zero-ring and similarly as above, $I \in S$. The result follows.

COROLlary 3 [3]. If a class $M$ is hereditary and $M \subseteq M^{0}$ then the radical $S M$ is hereditary.

Proof. If $A \in S M$ is a zero-ring then by [1, Theorem 2] $A \in L M$, so by Corollary 2, $A \in \overline{S M}$. Now Corollary 1 and Proposition 1 imply that $\overline{S M}$ is a left strong radical. But $M=\bar{M} \subseteq \overline{S M}$, so $S M=\overline{S M}$.

As there are supernilpotent hereditary radicals that are not left strong (for example the Brown-McCoy radical [1]) then the condition (a) of Proposition 1 is essential. Now we prove that neither condition (b) nor condition (c) can be dropped.

EXAMPLe 1. Let $\mathbf{Q}$ be the field of rational numbers and let $R$ be the ring of matrices of the form $\left(\begin{array}{ll}a & b \\ 0 & 0\end{array}\right)$ where $a, b \in \mathbf{Q}$. Then $L=\left\{\left(\begin{array}{ll}a & 0 \\ 0 & 0\end{array}\right) \mid a \in \mathbf{Q}\right\}$ is a left ideal of $R$ isomorphic to $\mathbf{Q}$ such that $L^{*}=R$ and $I=\left\{\left(\begin{array}{ll}0 & b \\ 0 & 0\end{array}\right) \mid b \in \mathbf{Q}\right\}$ is an ideal of $R$ isomorphic with $\mathbf{Q}^{0}$.

If $M=\{\mathbf{Q}\}$ then $L M$ does not contain non-zero zero-rings. Tl:us by [1, Theorem 2] $S M$ also does not contain non-zero zero-rings. Hence $S M$ satisfies (a) and (c). Also $L \in \overline{S M}$ but $R \notin \overline{S M}$, so $\overline{S M}$ is not left strong.

Now let $M=\left\{\mathbf{Q}, \mathbf{Q}^{\circ}\right\}$. Then, since the class $D$ of all divisible rings is a left strong radical, and $M \subseteq D$, we have $S M \subseteq D$. Thus $\mathbf{Q}^{0} \notin \overline{S M}$ and also $R \notin \overline{S M}$ but $L \in \overline{S M}$. The radical $S M$, however, satisfies (a) and, by Corollary 1 , (b).

Now we prove

Proposition 2. If a radical $S$ has the properties (a) $S$ is left stable, (b) $S \subseteq S^{0}$, and (c) any zero-ring of $S$ is in $\bar{S}$, then $\bar{S}$ is left stable.

Proof. Let $A$ be a ring and $L \in \bar{S}$ a left ideal of a left ideal $K$ of $A$. We need to show that if $I$ is an ideal of $L^{*}$ then $I \in S$. Without loss of generality we may assume that $K=A^{1} L$. Let us first make the following observation: (*) if $R$ is a subring of $L^{*}$ such that $R^{2} \subseteq S(I)$ then $R \in S$. Indeed, $(R+S(I)) / S(I)$ is isomorphic to some ideal of $\left(L^{*} / S(I)\right)^{0} \approx$ $\left(L^{*}\right)^{0} /(S(I))^{0}$, so by Lemma 1 and conditions (a), (c), $(R+S(I)) / S(I) \in S$. Thus $R \in S$. Now $I K L$ is a left ideal of $I K$ and $I K$ is a left ideal of $I$. Thus condition (a) implies that 
$I K L \subseteq S(I)$. Now $(L I K)^{2}=L I K L I K \subseteq L S(I) I K \subseteq S(I)$, so by $(*) L I K \in S$. But $L I K$ is a left ideal of $I K$ and $I K$ is a left ideal of $I$. Thus $L I K \subseteq S(I)$. This implies that $I^{*} L^{*} I^{*} I K^{*} I^{*}=$ $I^{*} A^{1} L A^{1} I^{*} I K A^{1} I^{*}=I^{*} L I^{*} I K I^{*} \subseteq I^{*} L I K I^{*} \subseteq I^{*} S(I) I^{*} \subseteq S(I)$ (here $I^{*}$ denotes the ideal of $A$ generated by $I$; of course $I$ is an ideal of $\left.I^{*}\right)$. In particular $I^{6} \subseteq S(I)$. By $(*), I \in S$ and the result follows.

Similarly as in Example 1 one can show that none of the conditions of Proposition 2 can be dropped. As in Corollary 3 we check.

COROLlary 4 [4]. The lower left stable radical determined by a hereditary class $M$ such that $M \subseteq M^{0}$ is hereditary.

In [8] Stewart has called a radical $S$ strict if for any subring $B$ of a ring $A$ the fact that $B \in S$ implies $B^{*} \in S$. Of course any strict radical is left and right stable and for any class $M$ there exists the lower strict radical determined by $M$ i.e. the smallest strict radical containing $M$. In view of the results of this section it is natural to ask whether $M$ is a hereditary class such that $M \subseteq M^{0}$ then the lower strict radical determined by $M$ is also hereditary.

ExAmple 2. Let $M=\left\{K, K^{0}\right\}$ where $K$ is the field of two elements and let $S$ be the lower strict radical determined by $M$. Then the ring $K[x]$ of polynomials over $K$ of indeterminate $x$ is in $S$. By [8, Theorem 2.1] it is easy to see that $U=\{A \mid$ any non-zero homomorphic image of $A$ contains non-zero idempotents or nilpotents $\}$ is a strict radical. Since $M \subseteq U$ then $S \subseteq U$. This shows that the ideal $(x)$ of $K[x]$ generated by $x$ is not $S$-radical. Therefore $S$ is not hereditary even though $M \subseteq M^{0}$ and $M$ is hereditary with respect to subrings.

2. One-sided hereditariness. It has been shown in [4] that there exists a class $M$ hereditary with respect to subrings such that $M \subseteq M^{0}$ and such that $S t M$ is neither left nor right hereditary. Now we shall investigate one-sided hereditariness of the lower strong radicals. First we make some observations on such radicals.

LEMMA 2. For any element $a$ of $a$ ring $R, I=r(a) a$, where $r(a)=\{x \in R \mid a x=0\}$, is an ideal of $R a$ and $I^{2}=0$. In addition $R a / I$ is a homomorphic image of $a R$.

Proof. The first part of the lemma is clear. It is also easy to see that the mapping $f: a R \rightarrow R a / I$ given by $f(a x)=x a+I$ is well defined. Clearly $f$ is onto and preserves addition. Let $x, y \in R$. Then $f($ axay $)=x a y a+I=(x a+I)(y a+I)=f(a x) \cdot f(a y)$, so $f$ also preserves multiplication.

A radical is said [6] to be principally left (right) hereditary if for every radical ring $R$ and element $a \in R R a(a R)$ is radical.

By Lemma 2 we obtain immediately a result related to the question of $[6, p .363]$.

COROLLARY 5. If a radical $S$ contains all zero-rings then $S$ is principally left hereditary if and only if $S$ is principally right hereditary.

By [2, Lemma 3] we know that if $S \neq 0$ is a left principally hereditary and right strong 
radical in the class of all associative algebras over a field $F$ then $S$ contains all zero- $F$-algebras. Thus the quoted question of Sands has a positive answer for radicals in the class of $F$-algebras.

Another result concerning similar questions and related to Sands question of $[7, \mathrm{p}$. $23]$ is the following.

COROLlaRY 6. Any right hereditary radical is left principally hereditary.

Proof. By Lemma 2 it is enough to prove that if $R \in S$ and $P$ is a zero-subring of $R$ then $P \in S$. But since $P$ is a zero-ring then it is a right ideal of $P R^{1}$. Thus right hereditariness of $S$ implies $P \in S$.

Using Lemma 2 we can also give another proof of the following result.

COROLLARY 7 [7]. Any left strong and right hereditary radical $S$ is right strong and left hereditary.

Proof. Let $L$ be a left ideal of $R \in S$. Then by Corollary $6 R a \in S$ for any $a \in L$. Using the left strongness of $S$ we get $S(L) \supseteq \sum_{a \in L} R a=R L$. Now $L / R L=(L / R L)^{\mathrm{o}} \approx L^{\mathrm{o}} /(R L)^{\mathrm{o}}$ is an ideal of $R^{0} /(R L)^{0}$. But by [7, Lemma 1$], R^{0} \in S$. Thus $L / R L \in S$ and in consequence $L \in S$.

Now let $P \in S$ be a right ideal of a ring $A$. By [7, Lemma 1$]\left(P^{*}\right)^{0} \in S$. If $a \in P$ then $a \cdot P^{*}$ is a right ideal of $P$. Since $(I a)^{2}=0$ where $I=\left\{x \in P^{*} \mid a x=0\right\}$ then $I a$ is isomorphic to some ideal of $\left(P^{*}\right)^{0}$. Thus the right hereditariness of $S$ implies $a P^{*} \in S$ and $I a \in S$. Now Lemma 2 yields $P^{*} a \in S$. This and the left strongness of $S$ implies that $P^{*} P=\sum_{a \in P} P^{*} a \subseteq$
$S\left(P^{*}\right)$. As in the first part we now obtain $P^{*} \in S$. This ends the proof.

A left and right hereditary class of rings will be called one-sided hereditary.

For any class $M$ define $\tilde{M}=\{A \mid$ any one-sided ideal of $A$ is in $M\}$. It is easy to see that if $M$ is a radical then so is $\tilde{M}$. Now we prove

Proposition 3. If a radical $S$ has the properties (a) $S$ is left strong, (b) $S \subseteq S^{0}$, and (c) any ideal of a zero-ring of $S$ is in $\tilde{S}$, then $\tilde{S}$ is left strong.

Proof. Let $L \in \tilde{S}$ be a left ideal of a ring $A$ and let $K$ be a left ideal of $L^{*}$. Then for any $k \in K, k L$ is a right ideal of $L \in \tilde{S}$, so $k L \in S$. By Lemma $2,(L k+r(a) k) / r(a) k$ is a homomorphic image of $k L$, hence $(L k+r(a) k) / r(a) k \in S$. But $r(a) k$ is isomorphic to an ideal of $\left(L^{*}\right)^{0}$, so $r(a) k \in S$. Thus $L k \in S$. This and the fact that $L k$ is a left ideal of $K$ implies $L K=\sum_{k \in K} L k \subseteq S(K)$. Hence $K^{3} \subseteq\left(L^{*} K\right) K=\left(L A^{1} K\right) K \subseteq L A^{1} K^{2} \subseteq L L^{*} K \subseteq$ $L K \subseteq S(K)$. But $K / K^{2} \approx K^{0} /\left(K^{2}\right)^{0} \in S$ and $K^{2} / K^{3} \approx\left(K^{2}\right)^{0} /\left(K^{3}\right)^{0} \in S$, so $K / K^{3} \in S$. Therefore $K \in S$.

Now let $P$ be a right ideal in $L^{*}$. Let us observe that the set $\left(\begin{array}{cc}P & P \\ \left(L^{*}\right)^{1} & L^{*}\end{array}\right)$ of matrices with the natural operations is a ring and $\left(\begin{array}{cc}0 & P L \\ 0 & L\end{array}\right)$ is its left ideal. Certainly $\left(\begin{array}{ll}0 & 0 \\ 0 & L\end{array}\right) \approx L \epsilon$ $\tilde{S}$. In addition $\left(\begin{array}{cc}0 & P L \\ 0 & 0\end{array}\right)$ is a zero-ring which is a sum of its subrings which are homomor- 
phic images of $L^{0}$, so by (b), (c) $\left(\begin{array}{cc}0 & P L \\ 0 & 0\end{array}\right) \in \tilde{S}$. Thus $\left(\begin{array}{cc}0 & P L \\ 0 & L\end{array}\right) \in \tilde{S}$ and by the already proved result every left ideal of $\left(\begin{array}{cc}0 & P L \\ 0 & L\end{array}\right)+\left(\begin{array}{cc}0 & P L \\ 0 & L\end{array}\right)\left(\begin{array}{cc}P & P \\ \left(L^{*}\right)^{1} & L^{*}\end{array}\right)$ is in $S$. Hence $\left(\begin{array}{cc}P L\left(L^{*}\right)^{1} & 0 \\ L\left(L^{*}\right)^{1} & 0\end{array}\right) \in$ $S$. This shows that if $L^{* *}$ denotes the ideal of $L^{*}$ generated by $L$ then $\left(P \cap L^{* *}\right)^{2} \subseteq$ $P L^{* *}=P L\left(L^{*}\right)^{1} \in S$. By our assumptions $\left(P \cap L^{* *}\right)^{0} \dot{\in} S$. This and the fact that $P \cap L^{* *} / P L^{* *}$ is a zero-ring implies $P \cap L^{* *} / P L^{* *} \in S$. Hence $P \cap L^{* *} \in S$. Now $L^{*} / L^{* *}$ is a zero-ring, so every subring of it is in $S$. In particular $P+L^{* *} / L^{* *} \in S$. Thus $P \in S$. The result follows.

Similarly to Corollary 3 one can check

COROLlary 8. If $M$ is a one-sided hereditary class such that $M \subseteq M^{0}$ then the lower left strong radical determined by $M$ is one-sided hereditary.

Example 3. Let $K$ be the field of two elements and let $S$ be the lower left strong radical determined by $M=\left\{K, K^{0}\right\}$. It is easy to see that the ring $R$ of all $2 \times 2$ matrices over $K$ is in $S$ and that the field $F$ of four elements can be embedded into $R$. But by [1, Theorem 2] $F \notin S$. This shows that $S$ is not hereditary with respect to subrings even though $M$ is such and $M \subseteq M^{0}$.

All obtained results concerning hereditariness of lower radicals can be demonstrated by the following table (the sign "+" means that the corresponding radical satisfies the condition which was satisfied by $M$ and "-" that it does not. $M$ denotes a class such that $M \subseteq M^{0}$ ).

\begin{tabular}{lcccc}
\hline Radicals & lower & $\begin{array}{c}\text { lower } \\
\text { left strong }\end{array}$ & $\begin{array}{c}\text { lower } \\
\text { left stable }\end{array}$ & $\begin{array}{c}\text { lower } \\
\text { strict }\end{array}$ \\
\hline $\begin{array}{l}\text { hereditary } \\
\text { one-sided } \\
\text { hereditary } \\
\text { hereditary } \\
\text { with respect } \\
\text { to subrings }\end{array}$ & + & + & + & - \\
\hline
\end{tabular}

Proposition 4. If the lower left strong radical SM determined by $M$ is hereditary with respect to subrings then $M$ contains a finite field or all SM-rings are nil.

Proof. Let $R \in S M$ and $\langle x\rangle$ denote the subring of $R$ generated by $x \in R$. Then it is well known that $x$ is nilpotent or $\langle x\rangle$ can be homomorphically mapped onto a finite field $F$. By [1, Theorem 2] $F \in M$. This proves the proposition.

The famous problem of Koethe asks whether every nil left ideal of a ring $R$ is contained in the nil radical of $R$, i.e. whether the nil radical is left strong. By Proposition 4 we have

COROLlary 9. The lower left strong radical determined by the class of all nil rings is hereditary with respect to subrings if and only if the Koethe problem has a positive solution. 
3. Lattice of strong hereditary radicals. In this section we shall prove

Proposirion 5. If $\left\{S_{i}\right\}$ is a family of left strong and hereditary radicals then so is $S=L\left(\cup S_{i}\right)$.

Proof. By Corollary $2 S$ is hereditary, so we shall prove that $S$ is strong. Let $L$ be a left $S$-ideal of an $S$-semisimple ring $R$ and let $I=\{a \in L \mid L a=0\}$. If $S(L / I) \neq 0$ then for some $i, S_{i}(L / I)=J / I \neq 0$. Let us observe that $(L / I)$. $(J / I)$ is an ideal of $J / I \in S_{i}$, so the hereditariness of $S_{i}$ implies $(L / I)$. $(J / I) \in S_{i}$. But $(L / I)$. $(J / I)=(L J+I) / I \approx L J /(L J \cap I)$. Now for any $b \in L$ the mapping $f_{b}:(J / I)^{0} \rightarrow(b J)^{0}$ given by $f_{b}(j+I)=b j$ is a well defined (as $L I=0$ ) ring homomorphism. Hence, since $S_{i} \subseteq S_{i}^{0}[7],(b J)^{0} \in S_{i}$. Thus $(L J)^{0}=\sum_{b \in L}(b J)^{0} \in S_{i}$. But $S_{i}$ is hereditary and $I^{2}=0$, so $L J \cap I \approx(L J)^{0} \cap I^{0} \in S$. Hence $L J$ is a left $S_{i}$-ideal of $R$. Thus $L J \subseteq S_{i}(R) \subseteq S(R)=0$, so $J=I$. This contradiction shows that $I=L$ and hence $L^{2}=0$. But then $\left(L^{*}\right)^{2}=(L+L R)^{2}=0$. Therefore $L \in S$ is an ideal of $L^{*}$, so $L \subseteq S(R)=0$. This proves the proposition.

COROLlary 10. The class of all left strong and hereditary radicals is a complete sublattice in the lattice of all radicals.

\section{REFERENCES}

1. N. Divinsky, J. Krempa and A. Suliński, Strong radical properties of alternative and associative rings, J. Algebra 17 (1971), 369-388.

2. M. Jaegerman and A. D. Sands, On normal radicals, $N$-radicals and A-radicals, J. Algebra 50 (1978), 337-349.

3. E. R. Puczyłowski, On lower strong radicals in alternative algebras, Bull. Acad. Polon. Sci. 25 (1977), 617-622.

4. E. R. Puczyłowski, Remarks on stable radicals, Bull. Acad. Polon. Sci. 28 (1980), 11-16.

5. R. F. Rossa and R. L. Tangeman, General hereditary for radical theory, Proc. Edinburgh Math. Soc. 20 (1977), 333-337.

6. A. D. Sands, On normal radicals, J. London Math. Soc. 11 (1975), 361-365.

7. A. D. Sands, On relations among radical properties, Glasgow Math. J. 18 (1977), 17-23.

8. P. N. Stewart, Strict radical classes of associative rings, Proc. Amer. Math. Soc. 39 (1973), 273-278.

9. R. Wiegandt, Radical and semisimple classes of rings (Ontario, 1974).

INSTITUTE OF MATHEMATICS

UNIVERSITY OF WARSAW

PKIN, 00-901 WaRSAW 